\title{
Extraction of state-to-state reactive scattering attributes from wave packet in reactant Jacobi coordinates
}

\author{
Zhigang Sun, ${ }^{1,2}$ Hua Guo, ${ }^{3, a)}$ and Dong H. Zhang ${ }^{1, a)}$ \\ ${ }^{1}$ State Key Laboratory of Molecular Reaction Dynamics and Center for Theoretical and Computational \\ Chemistry, Dalian Institute of Chemical Physics, Chinese Academy of Sciences, 116023 Dalian, \\ People's Republic of China \\ ${ }^{2}$ Department of Chemistry, Duke University, Durham, North Carolina 27708, USA \\ ${ }^{3}$ Department of Chemistry and Chemical Biology, University of New Mexico, Albuquerque, \\ New Mexico 87131, USA
}

(Received 18 December 2009; accepted 2 February 2010; published online 25 February 2010)

\begin{abstract}
The $S$-matrix for a scattering system provides the most detailed information about the dynamics. In this work, we discuss the calculation of $S$-matrix elements for the $\mathrm{A}+\mathrm{BC} \rightarrow \mathrm{AB}+\mathrm{C}, \mathrm{AC}+\mathrm{B}$ type reaction. Two methods for extracting $S$-matrix elements from a single wave packet in reactant Jacobi coordinates are reviewed and compared. Both methods are capable of extracting the state-to-state attributes for both product channels from a single wave packet propagation. It is shown through the examples of $\mathrm{H}+\mathrm{HD}, \mathrm{Cl}+\mathrm{H}_{2}$, and $\mathrm{H}+\mathrm{HCl}$ reactions that such reactant coordinate based methods are easy to implement, numerically efficient, and accurate. Additional efficiency can be gained by the use of a $L$-shaped grid with two-dimensional fast Fourier transform. (C) 2010 American Institute of Physics. [doi:10.1063/1.3328109]
\end{abstract}

\section{INTRODUCTION}

Since the establishment of quantum theory for atomdiatom reactive scattering in $1970 \mathrm{~s},{ }^{1}$ significant progress has been made, particularly in the past two decades, with the development of hyperspherical coordinate based timeindependent coupled-channel (TID-CC) method and Jacobi coordinate based time-dependent wave packet (TDWP) method. ${ }^{2}$ The TID-CC method has particular advantages for treating systems requiring relative small numbers of basis functions and/or collisions with very low kinetic energy. It can now be routinely applied to study light triatomic systems, such as $\mathrm{H}+\mathrm{H}_{2}, \mathrm{~F}+\mathrm{H}_{2}$, and $\mathrm{Cl}+\mathrm{H}_{2}$ reactions and their isotopically substituted analogs, ${ }^{3,4}$ and has provided valuable insights into chemical reaction dynamics. Recently, the TID-CC method has been extended to calculate fully converged state-to-state differential cross sections (DCS) for insertion reactions such as the $\mathrm{O}+\mathrm{H}_{2}, \mathrm{C}+\mathrm{H}_{2}, \mathrm{~S}+\mathrm{H}_{2}$, and $\mathrm{N}$ $+\mathrm{H}_{2}$ reactions. These results have served as accurate benchmarks for dynamical studies of complex-forming reactions, featuring barrierless reaction pathways through deep potential wells. ${ }^{5-9}$

However, due to the $N^{3}$ ( $N$ is the number of basis functions) scaling law in the formalism, it is extremely difficult to extend the TID-CC method to calculate cross sections for more complex systems, such the $\mathrm{H}+\mathrm{O}_{2}$ reaction, which possesses a deep well and two heavy (nonhydrogen) atoms. ${ }^{10}$ On the other hand, the TDWP method scales more favorably than the TID-CC method in both memory and the number of arithmetic operations because the basic operation involves matrix-vector multiplication. As a result, it is much more

\footnotetext{
${ }^{a)}$ Authors to whom correspondence should be addressed. Electronic addresses: hguo@unm.edu and zhangdh@dicp.ac.cn.
}

suitable for complex systems requiring a large number of basis functions. It has been shown that the TDWP approach is particularly powerful for calculating initial state selected total reaction probabilities for systems involving three or more atoms by using reactant Jacobi coordinates. ${ }^{11-13}$ However, to extract $S$-matrix elements in a TDWP calculation, traditionally one needs to transform the wave function initially prepared in reactant Jacobi coordinates to product Jacobi coordinates, making the calculations cumbersome and potentially less accurate. ${ }^{14-19}$ In the following discussion, we will call this approach the product coordinate based (PCB) method.

Recently, we have put forth a reactant coordinate based (RCB) wave packet method to extract product state-resolved attributes in both product channels. ${ }^{20-23}$ Taking advantage of a $L$-shaped grid to propagate in reactant coordinates, this collocation method is efficient and accurate and has been successfully applied to calculate state-to-state DCSs for the $\mathrm{H}+\mathrm{HD}, \mathrm{F}+\mathrm{H}_{2}, \mathrm{H}+\mathrm{NH}, \mathrm{H}+\mathrm{ND},{ }^{24} \mathrm{H}+\mathrm{O}_{2},{ }^{20,21}$ and $\mathrm{O}+\mathrm{O}_{2}$ reactions. ${ }^{25}$ The $L$-shaped grid method saves a large number of grid points, as compared with a conventional rectangular grid, which makes this RCB wave packet method particularly attractive and presents an efficient alternative to the PCB approaches. ${ }^{14-16}$

In 2006, Gómez-Carrasco and Roncero ${ }^{26}$ developed an coordinate transform method to extract state-to-state information in one product channel, ${ }^{27}$ also from the TDWP propagated in reactant Jacobi coordinates. By careful numerical testing with several reactive scattering systems, these authors reached a conclusion that their RCB method is generally more efficient than the PCB method, especially for $J>0$ cases. Different from our approach, however, a rectangular grid was used in their calculations.

In this paper, we first compare the two RCB wave packet 
methods, introduced by $\mathrm{us}^{20}$ and by Gómez-Carrasco and Roncero, ${ }^{26}$ for studying the $\mathrm{A}+\mathrm{BC} \rightarrow \mathrm{AB}+\mathrm{C}, \mathrm{AC}+\mathrm{B}$ type reaction at the state-to-state level, using the $\mathrm{H}+\mathrm{HD}$ reaction as an example. These RCB methods were implemented with the efficient and accurate Chebyshev propagator. ${ }^{28-30}$ It is shown that the coordinate transform RCB method can also be used to extract $S$-matrix elements in both product channels with a single propagation, as the RCB method introduced by us ${ }^{20,22}$ and it is generally more efficient.

The two RCB methods were then applied to the $\mathrm{Cl}+\mathrm{H}_{2}$ reaction to demonstrate the advantages of a $L$-shaped grid. Such a grid saves computational time, especially when the long-range potential in the reactant channel is important. Finally, it is also shown that the extraction of the final product states-resolved attributes does not require placing the projection plane in the "true" asymptote, provided that the finalstate interaction is small. This point is illustrated in the calculation of state-to-state DCSs for both product channels of the $\mathrm{H}+\mathrm{HCl}$ reaction. Such savings make the RCB approach a method of choice for state-to-state cross-section calculations for many reactive scattering systems.

This publication is arranged as follows. In Sec. II, the basic theoretical aspects for extracting state-to-state cross sections in both product channels using the RCB methods are presented in detail. In Sec. III, the state-to-state cross sections for the $\mathrm{H}+\mathrm{HD}\left(v_{0}=0, j_{0}=1\right)$ reaction calculated by both RCB methods are first compared. The power of the combining the RCB method with a $L$-shaped grid is then demonstrated for the $\mathrm{Cl}+\mathrm{H}_{2}\left(v_{0}=0, j_{0}=0\right)$ and $\mathrm{H}+\mathrm{HCl}\left(v_{0}\right.$ $\left.=0, j_{0}=0\right)$ reactions. Finally, Sec. IV concludes.

\section{THEORY}

\section{A. Hamiltonian and discretization}

To study the $\mathrm{A}+\mathrm{BC} \rightarrow \mathrm{AB}+\mathrm{C}, \mathrm{AC}+\mathrm{B}$ type reaction at the state-to-state level, we use three sets of body-fixed (BF) Jacobi coordinates to represent the corresponding wave functions: one for reactants $\mathrm{A}+\mathrm{BC}$ (denoted as $\alpha$ arrangement), the other for products $\mathrm{AB}+\mathrm{C}$ (denoted as $\beta$ arrangement), and the last for products $\mathrm{AC}+\mathrm{B}$ (denoted as $\gamma$ arrangement). Following the usual definition, ${ }^{1,31}$ we write the coordinates as $\left(R_{\nu}, r_{\nu}, \theta_{\nu} ; \boldsymbol{\Omega}_{\nu}\right)$, where $\nu$ is either $\alpha, \beta$, or $\gamma$. For $\nu=\alpha(\nu$ $=\beta, \gamma), r_{\nu}$ is the $\mathrm{BC}(\mathrm{AB}, \mathrm{AC})$ bond length, $R_{\nu}$ is the length of the vector $\hat{R}_{\nu}$ pointing from $\mathrm{A}(\mathrm{C}, \mathrm{B})$ to the $\mathrm{BC}(\mathrm{AB}, \mathrm{AC})$ center of mass, and $\theta_{\nu}$ is the angle between the $\mathrm{BC}(\mathrm{AB}, \mathrm{AC})$ bond and $\hat{R}_{\nu} ; \boldsymbol{\Omega}_{\nu}$ denotes the Euler angles orienting $\hat{R}_{\nu}$ in the space-fixed $(\mathrm{SF})$ frame.

In this work, the propagation of the wave packet is carried out exclusively in reactant Jacobi coordinates ${ }^{20,22}$ in the $\mathrm{BF}$ frame using the Chebyshev real wave packet (CRWP) approach. ${ }^{15,28,29,32,33}$ The corresponding Hamiltonian for a given total angular momentum $J$ can be written as

$$
\hat{H}=-\frac{\hbar^{2}}{2 \mu_{R_{\alpha}}} \frac{\partial^{2}}{\partial R_{\alpha}^{2}}-\frac{\hbar^{2}}{2 \mu_{r_{\alpha}}} \frac{\partial^{2}}{\partial r_{\alpha}^{2}}+\frac{(\boldsymbol{J}-\boldsymbol{j})^{2}}{2 \mu_{R_{\alpha}} R_{\alpha}^{2}}+\frac{j^{2}}{2 \mu_{r_{\alpha}} r_{\alpha}^{2}}+V
$$

where $\mu_{r_{\alpha}}$ is the reduced mass of $\mathrm{BC}$ and $\mu_{R_{\alpha}}$ is the reduced mass between the center of mass of $\mathrm{A}$ and $\mathrm{BC}, \boldsymbol{J}$ is the total angular momentum operator, and $\boldsymbol{j}$ is the rotational angular momentum operator of BC.

Following our previous studies, the Hamiltonian and wave packet were discretized in a mixed representation. A direct product discrete variable representation (DVR) is used for the two radial degrees of freedom and a finite basis representation $(\mathrm{FBR})$ of spherical harmonics basis $y_{j K}\left(\theta_{\alpha}\right)$ is used for the angular degree of freedom. ${ }^{34}$ Thus the wave function in the BF frame can be written as ${ }^{20,35-38}$

$$
\Psi^{J M \epsilon}\left(\boldsymbol{R}_{\alpha}, \boldsymbol{r}_{\alpha}\right)=\sum_{K_{\alpha}} \mathcal{D}_{M K_{\alpha}}^{J \epsilon^{*}}\left(\boldsymbol{\Omega}_{\alpha}\right) \psi_{\alpha}\left(R_{\alpha}, r_{\alpha}, \theta_{\alpha}^{K_{\alpha}} ; K_{\alpha}\right),
$$

where $\mathcal{D}_{M K_{\alpha}}^{J \epsilon^{*}}\left(\boldsymbol{\Omega}_{\alpha}\right)$ is the parity-adapted normalized rotation matrix, depending only on the Euler angles $\boldsymbol{\Omega}_{\alpha}$,

$$
\begin{aligned}
\mathcal{D}_{M K_{\alpha}}^{J \epsilon^{*}}\left(\boldsymbol{\Omega}_{\alpha}\right)= & \left(1+\delta_{K_{\alpha} 0}\right)^{-1 / 2} \sqrt{\frac{2 J+1}{8 \pi}}\left[D_{M K_{\alpha}}^{J *}\left(\boldsymbol{\Omega}_{\alpha}\right)\right. \\
& \left.+\epsilon(-1)^{J+K_{\alpha}} D_{M-K_{\alpha}}^{J *}\left(\boldsymbol{\Omega}_{\alpha}\right)\right]
\end{aligned}
$$

where $\epsilon$ is the parity of the system defined as $\epsilon=(-1)^{j+l}$ with $l$ being the orbital angular momentum quantum number, and $D_{M K}^{J}(\boldsymbol{\Omega})$ is Wigner rotation matrix. ${ }^{39}$ In Eq. (2), $\psi_{\alpha}\left(R_{\alpha}, r_{\alpha}, \theta_{\alpha} ; K_{\alpha}\right)$, which only depends on three internal coordinates of the system and the projection $\left(K_{\alpha}\right)$ of total angular momentum on the BF $z$ axis (along $\boldsymbol{R}_{\alpha}$ ), can be expanded as

$$
\psi_{\alpha}\left(R_{\alpha}, r_{\alpha}, \theta_{\alpha}^{K_{\alpha}} ; K_{\alpha}\right)=\sum_{n, m, j} F_{n m j}^{K_{\alpha}} u_{n}\left(R_{\alpha}\right) \phi_{m}\left(r_{\alpha}\right) y_{j K_{\alpha}}\left(\theta_{\alpha}\right),
$$

where $n$ and $m$ are the radial basis labels, $u_{n}\left(R_{\alpha}\right)$ and $\phi_{m}\left(r_{\alpha}\right)$ are the radial basis functions for $R$ and $r$, respectively, and $y_{j K_{\alpha}}=\sqrt{[(2 j+1) /(4 \pi)]} d_{K_{\alpha}{ }^{0}}$ are spherical harmonics. $d_{K_{\alpha} K_{\nu}}$ is a reduced Wigner rotation matrix. ${ }^{39}$

\section{B. Initial wave packet}

In a wave packet calculation, the initial condition of the wave packet has to be set up before its propagation. For atom-diatom scattering, the initial wave packet in the SF frame $\left(v_{0}, j_{0}, l_{0}\right)$ (Refs. 15, 20, and 40) can be constructed simply as

$$
\Psi_{\alpha v_{0} j_{0} l_{0}}^{J M \epsilon}=G\left(R_{\alpha}\right) \phi_{v_{0} j_{0}}\left(r_{\alpha}\right)\left|J M j_{0} l_{0} \epsilon\right\rangle
$$

where $\left|J M j_{0} l_{0} \epsilon\right\rangle$ is the total angular momentum eigenfunction in the SF representation with parity of system $\epsilon=(-1)^{j_{0}+l_{0}}, \phi_{v_{0} j_{0}}\left(r_{\alpha}\right)$ is the rovibrational eigenfunction for molecule BC, and $G\left(R_{\alpha}\right)$ is a Gaussian wave packet defined as

$$
G\left(R_{\alpha}\right)=\exp \left[-\frac{\left(R_{\alpha}-R_{\alpha}^{c}\right)^{2}}{\tau^{2}}\right] \cos \left(k_{c} R_{\alpha}\right),
$$

where $k_{c}$ is the translational momentum.

In order to propagate the wave packet in the BF frame, one should transform $\left|J M j_{0} l_{0} \epsilon\right\rangle$ in Eq. (5) to its BF counterpart as ${ }^{41}$ 


$$
\begin{aligned}
\left|J M j_{0} l_{0}\right\rangle & =\sum_{K_{\alpha} \geq 0} \boldsymbol{C}_{l_{0} K_{\alpha}}^{J j_{0} \epsilon}\left|J M j_{0} K_{\alpha}\right\rangle \\
& =\sum_{K_{\alpha} \geq 0} \boldsymbol{C}_{l_{0} K_{\alpha}}^{J j_{0} \epsilon} \mathcal{D}_{M K_{\alpha}}^{J \epsilon *}\left(\boldsymbol{\Omega}_{\alpha}\right) y_{j_{0} K_{\alpha}},
\end{aligned}
$$

where $C_{l K}^{J j \epsilon}$ is the parity-adapted orthogonal transform matrix between the SF and BF frames ${ }^{1,15,42-44}$ and given by

$$
C_{l K}^{J j \epsilon}=\sqrt{\frac{(2 l+1)}{(2 J+1)}} \sqrt{\left(2-\delta_{K, 0}\right)}\langle j K l 0 \mid J K\rangle,
$$

where $\langle\ldots\rangle$ is the Clebsch-Gordan coefficient.

\section{Real Chebyshev wave packet on L-shaped grid}

In the CRWP approach, the wave packet is propagated using the following modified Chebyshev recursion relation: ${ }^{45,46}$

$$
\phi_{k+1}=D\left(2 \hat{H}_{\text {scaled }} \phi_{k}-D \phi_{k-1}\right),
$$

with $\phi_{1}=D \hat{H}_{\text {scaled }} \phi_{0}$ and $\phi_{0}=\Psi_{\alpha v_{0} j_{0} l_{0}}^{J M \epsilon}$ given in Eqs. (5) and (6). This choice of $\phi_{0}$ implies that the initial wave packet is real and it contains both incoming and outgoing waves. Upon propagation, half of the wave packet goes outwards and is damped by $D$ as discussed in a subsequent section while the other half goes inwards, leading to reaction. If the damping term $D$ is real, the wave packet will stay real. This real wave packet approach has some numerical advantages over the propagation of the complex wave packet in the time domain, as it reduces the central processing unit (CPU) and memory costs by half. ${ }^{32}$ In addition, the Chebyshev propagation is more accurate than time propagation because the former avoids interpolation errors. ${ }^{28}$ The scaled Hamiltonian $\hat{H}_{\text {scaled }}$ is defined as

$$
D(r)=\left\{\begin{array}{l}
\exp \left[-\Delta_{t} \cdot C_{a} \cdot\left(\frac{r-r_{a}}{r_{b}-r_{a}}\right)^{n}\right], \quad r_{a} \leq r \leq r_{b}, \\
\exp \left[-\Delta_{t} \cdot C_{b} \cdot\left(\frac{r-r_{b}}{r_{\text {end }}-r_{b}}\right)^{n}\right]+\exp \left[-\Delta_{t} \cdot C_{a}\right], \quad r_{b}<r \leq r_{\text {end }} .
\end{array}\right.
$$

$$
\hat{H}_{\text {scaled }}=a_{s} \hat{H}-b_{s},
$$

where $a_{s}=2 /\left(H_{\max }-H_{\min }\right)$ and $b_{s}=1+a_{s} H_{\min }$. The scaling of the Hamiltonian is necessary to maintain the stability of the Chebyshev propagation.

During the wave packet propagation, the fast sineFourier transform is employed to evaluate the action of the radial kinetic energy operators onto the wave packet on a $L$-shaped grid, ${ }^{47}$ which may save a considerable amount of the grid points in some systems. ${ }^{22}$ The approach is similar in spirit with our earlier work using diatomic vibrational basis. ${ }^{48,20}$ A similar $L$-shape grid has been used with bond coordinates in a state-to-state study of the $\mathrm{Li}+\mathrm{HF}$ reactive

The action of the angular kinetic operators on the wave packet can be evaluated straightforwardly with the angular FBR, but it complicates the evaluation of the action of the potential energy operator. The generalized DVR (Ref. 50) is used to solve this problem, which converts the wave packet from the angular FBR to a grid representation using

$$
T_{i j}^{K}=\sqrt{\omega_{i}} y_{j K}\left(\theta_{i}^{K}\right),
$$

where $i$ denotes the index of the quadrature points for the Jacobi angular coordinate and $\omega_{i}$ is the corresponding weight. $\theta_{i}^{K}$ and $T_{j i}^{K}$ is given by the eigenvalues and eigenvectors of the matrix

$$
Y_{j j^{\prime}}^{K}=\left\langle y_{j K}(\cos \theta)|\cos \theta| y_{j^{\prime} K}(\cos \theta)\right\rangle .
$$

\section{Scattering boundary conditions}

We use a damping function in the following form to avoid the wave packet in reactant coordinates from reaching the grid boundary along $r$, scattering process but with $J=0 .{ }^{49}$
The first part $r_{a} \leq r \leq r_{b}$ is designed for the product with low kinetic energies while the second part $r_{b}<r \leq r_{\text {end }}$ is used for the product with relative higher kinetic energies. We found that the damping function in this form works well for reactive scattering process, especially when there is a complicated kinetic energy structure. The damping term along $R$ has the following simple form:

$$
D(R)=\exp \left[-\Delta_{t} \cdot C^{\prime} \cdot\left(\frac{R-R_{a}}{R_{b}-R_{a}}\right)^{n^{\prime}}\right],
$$




\section{E. Extraction of S-matrix elements and state-to-state cross sections}

To extract the state-to-state information, we place a plane defined as $R_{\nu}=R_{\nu 0}$ for the $\nu$ th $(\nu=\beta$ and $\gamma)$ arrangement channel using the corresponding product Jacobi coordinates. The $k$ th Chebyshev propagation state on the projection plane, $\Psi_{v_{\nu} j_{\nu} K_{\nu}}^{J \epsilon}\left(k ; R_{\nu 0}\right)$, can be calculated on the potential optimized discrete variable representation (PODVR) and Gauss-Legendre quadrature grids by a collocation method ${ }^{51,19}$ or by the coordinate transformation method $^{26}$ at each propagation step. The energy resolved scattering wave function $\Phi_{v_{\nu} j_{\nu} K_{\nu}}^{J \epsilon}(E)$ in the BF frame can be obtained by a discrete Fourier transform,

$$
\Phi_{v_{\nu^{j}{ }_{\nu} K_{\nu}}^{J \epsilon}}^{J \epsilon}\left(E ; R_{\nu 0}\right)=\sum_{k}\left(2-\delta_{k, 0}\right) \exp (-i k \zeta) \Psi_{\nu_{\nu^{j} \nu_{\nu} K_{\nu}}^{J \epsilon}}^{\epsilon_{1}}\left(k ; R_{\nu 0}\right),
$$

where the Chebyshev angle $\zeta$ is given by $\zeta=\arccos E_{\text {scaled. }}$. $E_{\text {scaled }}=a_{s} E-b_{s}$, which is in the range of $[-1,1]$, is the properly scaled total energy $E .^{15}$

Similar to the construction of the initial wave packet, the BF scattering wave function for an initial state $\left(v_{0} j_{0} l_{0}\right)$ $\Phi_{v_{\nu} j_{v} K_{v} \leftarrow v_{0} j_{0} l_{0}}^{J \epsilon}(E)$ in Eq. (15) is transformed into the SF frame by an orthogonal transformation matrix given by Eq. (8),

$$
\Phi_{v_{\nu} \nu_{\nu} l_{\nu} \leftarrow v_{0} j_{0} l_{0}}^{J \epsilon}(E)=\sum_{K_{\nu}} C_{l_{\nu} K_{\nu}}^{J j_{\nu} \epsilon} \Phi_{\nu_{\nu} j_{\nu} K_{\nu} \leftarrow v_{0} j_{0} l_{0}}^{J \epsilon}(E) .
$$

With $\Phi_{v_{\nu} j_{\nu} l_{v}}^{J \epsilon}(E)$, the desired state-to-state scattering matrix $S_{v_{\nu} j_{\nu} l_{\nu} \leftarrow v_{0} j_{0} l_{0}}^{J \epsilon}(E)(\nu=\beta, \gamma)$ in the SF frame can then be obtained by imposing the asymptotic boundary conditions,

$$
\begin{aligned}
& \Phi_{v_{\nu} j_{\nu} l_{\nu} \leftarrow v_{0} j_{0} l_{0}}^{J \epsilon}\left(E ; R_{\nu 0}\right) \\
&=- \mathcal{A}(E)\left(\frac{2 \pi \hbar^{2} k_{v_{\nu} j_{\nu}}}{\mu_{R_{\nu}}}\right)^{1 / 2} S_{v_{\nu} j_{\nu} l_{\nu} \leftarrow v_{0} j_{0} l_{0}}^{J \epsilon}(E) \\
& \times \overline{\mathcal{H}}_{l_{\nu}}\left(k_{v_{\nu} j_{\nu}} R_{\nu 0}\right),
\end{aligned}
$$

where $\mathcal{A}(E)$ is given by

$$
\mathcal{A}(E)=\left(\frac{\mu_{R_{\alpha}}}{2 \pi \hbar^{2} k_{v_{0} j_{0}}}\right)^{1 / 2} \int \overline{\mathcal{H}}_{l_{0}}\left(k_{v_{0} j_{0}} R_{\alpha}\right) G\left(R_{\alpha}\right) d R_{\alpha},
$$

where $\overline{\mathcal{H}}_{l}$ is an outgoing Riccati-Hankel function.

Finally, the scattering matrix $S_{v_{\nu} j_{\nu} l_{v} \leftarrow v_{0} j_{0} l_{0}}^{J}(E)$ in the SF frame is transformed into the helicity representation by the standard transformation,

$$
\begin{aligned}
S_{v^{\prime} j^{\prime} K^{\prime} \leftarrow v j K}^{J}= & \sum_{l^{\prime} l} i^{l-l^{\prime}} \sqrt{\frac{2 l^{\prime}+1}{2 J+1}}\left\langle j^{\prime} K^{\prime} l^{\prime} 0 \mid J K^{\prime}\right\rangle \\
& \times S_{v^{\prime} j^{\prime} l^{\prime} \leftarrow v j l}^{J} \sqrt{\frac{2 l+1}{2 J+1}}\langle j K l 0 \mid J K\rangle .
\end{aligned}
$$

By substituting the scattering matrix $S_{v_{\nu} j_{y} K_{\nu} \leftarrow v_{0} j_{0} K_{0}}^{J \epsilon}(E)$ in the helicity representation into the standard formulas, we obtain the state-to-state integral cross section (ICS), ${ }^{43}$

$$
\sigma_{v_{\nu} j_{\nu} \leftarrow v_{0} j_{0}}=\frac{\pi}{\left(2 j_{0}+1\right) k_{v_{0} j_{0}}^{2} K_{\nu}} \sum_{K_{0}} \sum_{J}(2 J+1)\left|S_{v_{\nu} j_{\nu} K_{\nu} \leftarrow v_{0} j_{0} K_{0}}^{J}\right|^{2}
$$

and the state-to-state DCS, ${ }^{43}$

$$
\begin{aligned}
& \frac{d \sigma_{v_{\nu} j_{\nu} \leftarrow v_{0} j_{0}}(\vartheta, E)}{d \Omega} \\
& =\frac{1}{\left(2 j_{0}+1\right)} \sum_{K_{\nu}} \sum_{K_{0}} \mid \frac{1}{2 i k_{v_{0} j_{0}}^{2}} \sum_{J}(2 J+1) \\
& \quad \times\left. d_{K_{\nu} K_{0}}^{J}(\vartheta) S_{v_{\nu} j_{\nu} K_{\nu} \leftarrow v_{0} j_{0} K_{0}}^{J}\right|^{2}
\end{aligned}
$$

in which $\vartheta$ is the scattering angle between the incoming A $+\mathrm{BC}$ reactants and the scattered $\mathrm{AC}+\mathrm{B} / \mathrm{AB}+\mathrm{C}$ products.

\section{F. Final state projection in reactant coordinates \\ 1. Collocation RCB method}

As detailed in our previous study, ${ }^{20}$ we implement the collocation RCB method as follows:

(1) Select two projection planes in the product asymptotes at $R_{\beta}=R_{\beta 0}$ and $R_{\gamma}=R_{\gamma 0}$, which should be as close as possible to, but have no overlap with, the damping regions.

(2) At the projection planes $R_{\nu 0}(\nu=\beta, \gamma)$, construct the PODVR, ${ }^{11,52} r_{\nu i}\left(i=1, \ldots, N_{\nu}^{r}\right)$, and Gaussian-Legendre quadratures, $\theta_{\nu j}\left(j=1, \ldots, N_{\nu}^{\theta}\right)$ for $r_{\nu}$ and $\theta_{\nu}$, respectively, to represent scattering wave functions. Then convert these grid points $\left(R_{\nu 0}, r_{\nu i}, \theta_{\nu j}\right)$ to a corresponding grid in reactant Jacobi coordinates.

$$
\begin{aligned}
\left(R_{\alpha k}, r_{\alpha k}, \theta_{\alpha k}\right)= & \left(R_{\alpha k}\left(R_{\nu 0}, r_{\nu i}, \theta_{\nu j}\right), r_{\alpha k}\left(R_{\nu 0}, r_{\nu i}, \theta_{\nu j}\right),\right. \\
& \left.\theta_{\alpha k}\left(R_{\nu 0}, r_{\nu i}, \theta_{\nu j}\right)\right),
\end{aligned}
$$

where $k=1, \ldots, N_{\beta}^{r} \times N_{\beta}^{\theta}+N_{\gamma}^{r} \times N_{\gamma}^{\theta}$. Note that this new grid does not coincide with the grid for propagating the wave packet, although both are defined in reactant Jacobi coordinates.

(3) Propagate the wave packet in the reactant Jacobi coordinate grid and determine the corresponding values on the projection grid: $\left(R_{\alpha k}, r_{\alpha k}, \theta_{\alpha k}\right)$ using a collocation method. The energy-dependent scattering wave functions can be obtained from the wave packets via a Fourier transform. The $S$-matrix elements can thus be extracted from the overlaps between the scattering wave functions and the asymptotic states on the projection grid.

Comparing with the schemes proposed in our original work, ${ }^{20}$ step (4) is removed here. This is because practical experience indicates that the long-range potential in the product channel has usually a negligible effect on the product states-resolved cross sections (vide infra).

To obtain the energy-dependent scattering wave functions on the projection planes in the product channels, we 
first evaluate the wave packet given by Eq. (4) for every $K_{\alpha}$ component at all quadrature grid points $\left(R_{\nu 0}, r_{\nu i}, \theta_{\nu j}\right)$ given in Eq. (22) at every propagation step,

$$
\begin{aligned}
\psi\left(t, R_{\nu 0}, r_{\nu i}, \theta_{\nu j} ; K_{\alpha}\right)= & \frac{R_{\nu 0} r_{\nu i}}{R_{\alpha} r_{\alpha}} \psi_{\alpha}\left[k, R_{\alpha}\left(R_{\nu 0}, r_{\nu i}, \theta_{\nu j}\right),\right. \\
& \left.r_{\alpha}\left(R_{\nu 0}, r_{\nu i}, \theta_{\nu j}\right), \theta_{\alpha}\left(R_{\nu 0}, r_{\nu i}, \theta_{\nu j}\right) ; K_{\alpha}\right],
\end{aligned}
$$

where $\nu=\beta, \gamma$, and hereafter indicates $\beta$ or $\gamma$ unless specified.

Then we rotate the $\mathrm{BF} z$ axis from $\hat{R}_{\alpha}$ to $\hat{R}_{\nu}$ according to ${ }^{1}$

$$
\begin{aligned}
& \psi_{\nu}\left(k, R_{\nu 0}, r_{\nu i}, \theta_{\nu j} ; K_{\nu}\right) \\
& =\sum_{K_{\alpha}} \psi\left(t, R_{\nu 0}, r_{\nu i}, \theta_{\nu j} ; K_{\alpha}\right) \frac{1}{\sqrt{\left(1+\delta_{K_{\nu}, 0}\right)\left(1+\delta_{K_{\alpha} 0}\right)}} \\
& \quad \times\left[d_{K_{\nu}, K_{\alpha}}^{J}(\Delta)+\epsilon(-1)^{K_{\alpha}} d_{K_{\nu},-K_{\alpha}}^{J}(\Delta)\right],
\end{aligned}
$$

where $d_{K_{\nu} K_{\alpha}}^{J}(\Delta)$ is a reduced Wigner rotational matrix, ${ }^{39}$ and $\Delta$ is the angle between $\hat{R}_{\alpha}$ and $\hat{R}_{\nu}$.

Finally, we expand $\psi_{\nu}\left(R_{\nu 0}, r_{\nu i}, \theta_{\nu j} ; K_{\nu}\right)$ in terms of basis functions for $r_{\nu}$ by the collocation method ${ }^{51,19}$ and $\theta_{\nu}$ by Gauss-Legendre quadrature, and combine it with $\mathcal{D}_{M K_{\nu}}^{J \epsilon^{*}}\left(\boldsymbol{\Omega}_{\nu}\right)$ shown in Eq. (3) to get the wave packet on the project planes $R_{\nu}=R_{\nu 0}$

$$
\begin{aligned}
& \Psi_{v_{\nu} j_{\nu} K_{\nu}}^{J \epsilon}\left(k ; R_{\nu}=R_{\nu 0}\right) \\
& \quad=\sum_{K_{\nu}} \mathcal{D}_{M K_{\nu}}^{J \epsilon^{*}}\left(\boldsymbol{\Omega}_{\nu}\right) \sum_{v, j} C_{v j}^{K_{\nu}}(t) \phi_{v}\left(r_{\nu}\right) y_{j K_{\nu}}\left(\theta_{\nu}\right) .
\end{aligned}
$$

Typically, the CPU cost for projection is only about $20 \%$ of the total propagation time. In other words, with an additional 20\% of computational effort comparing with an initial state selected total reaction probability calculation in which only total reaction probabilities is calculated, one obtains a full column of the state-to-state $S$-matrix.

\section{Coordinate transform RCB method}

The coordinate transform method introduced by GómezCarrasco and Roncero ${ }^{26}$ has many similarities with the collocation RCB method. In both methods, the most timeconsuming part of the calculation, namely the propagation, is carried out in reactant coordinates. However, the former differs from the collocation RCB method in that the product states are represented on the reactant grid. The idea is based upon the fact that the product wave function $\psi_{\nu}\left(R_{\nu}, r_{\nu}, \theta_{\nu} ; K_{\nu}\right)$ has only two degrees of freedom, $r_{\nu}$ and $\theta_{\nu}$, since $R_{\nu}$ is fixed at the projection plane $R_{\nu 0}$. It is possible to design an "intermediate" coordinate system in which $R_{\nu}$ is combined with either $\left(R_{\alpha}, \theta_{\alpha}\right)$ or $\left(r_{\alpha}, \theta_{\alpha}\right)$ of the reactant coordinates to provide a convenient platform for projection. ${ }^{26}$ Conveniently, the grid points along reactant coordinates can be chosen as $\left(R_{\alpha i}, \theta_{\alpha j}\right)$ or $\left(r_{\alpha i}, \theta_{\alpha j}\right)$.

To implement this RCB method, the two-dimensional product wave functions are first transformed and stored in the reactant coordinate grid $\left(R_{\alpha i}, \theta_{\alpha j}\right)$ or $\left(r_{\alpha i}, \theta_{\alpha j}\right)$ before the propagation. In each propagation step, the wave packet is transformed to the same grid via two one-dimensional trans- formations, facilitating the calculation of the overlaps. This is a coordinate transform method because the transformation of the wave packet to $\left(R_{\alpha i}, \theta_{\alpha j}\right)$ or $\left(r_{\alpha i}, \theta_{\alpha j}\right)$ is simply the evaluation of the wave packet at $r_{i j}$ or $R_{i j}$, which corresponds to $R_{\nu 0}$. Instead of transforming the propagating wave packet from reactant coordinates to product coordinates in order to evaluating the product states-resolved information, it transforms the product state wave function from product coordinates to reactant coordinates. The computational costs are equivalent to those required for evaluating a norm. As the collocation RCB method, ${ }^{22,20}$ the coordinate transform RCB method is capable of evaluating states-resolved information of both product channels at the same time.

The choice between $\left(R_{\alpha i}, \theta_{\alpha j}\right)$ and $\left(r_{\alpha i}, \theta_{\alpha j}\right)$ for the intermediate coordinates depends on the skew angle $\omega$ for product channel $\beta$,

$$
\begin{aligned}
& \cos ^{2} \omega=a c \text { with } a=M_{C} /\left(M_{B}+M_{C}\right) \\
& \text { and } c=M_{A} /\left(M_{A}+M_{B}\right)
\end{aligned}
$$

and for product channel $\gamma$

$$
\begin{aligned}
& \cos ^{2} \omega=a c \text { with } a=M_{B} /\left(M_{B}+M_{C}\right) \\
& \text { and } c=M_{A} /\left(M_{A}+M_{C}\right)
\end{aligned}
$$

by assuming the grid spacings of $R_{\alpha}$ and $r_{\alpha}$ are comparable. The value $\pi / 2-\omega$ gives the angle between the product channel and reactant channel. Assuming we have similar grid spacings between $r_{\alpha}$ or $R_{\alpha},\left(r_{\alpha i}, \theta_{\alpha j}\right)$ are a more suitable choice for $\cos \omega<\sqrt{2} / 2$ and $\left(R_{\alpha i}, \theta_{\alpha j}\right)$ is preferred for $\cos \omega>\sqrt{2} / 2$. This is not difficult to understand. The variable of $R_{\alpha}$ or $r_{\alpha}$, which requires relatively sparser grid points to represent the product wave function, is always a better choice. In practical calculations, the grid spacings between $r_{\alpha}$ or $R_{\alpha}$ may have large difference and carefully testing is necessary for making the right choice.

Generally, the coordinate transform RCB method is more efficient than the collocation RCB method, if the quadrature points required for accurately propagating the wave packet are dense enough for extracting the product states-resolved information in the coordinate transform RCB method with high accuracy, as shown below. This is because that the grid for reactant coordinates must be dense enough to accurately represent the wave function of any arrangement channel. In fact, our experience indicates that the norm of the product wave function in the reactant coordinate grid provides a good criterion for checking the numerical convergence of the grid size used in the calculation.

The implementation of the coordinate transform RCB method has been discussed in detail by Gómez-Carrasco and Roncero. ${ }^{26}$ In our calculations, a more compact and efficient angular quadrature scheme is used, where different GaussLegendre quadrature points are adopted for different $K_{\alpha}$ values and the BF transform is carried out once after propagation. This $K_{\alpha}$-dependent quadrature method reduces the grid point number thus decreases memory requirement for the wave packet but increases memory requirement for potential, comparing with that the $K_{\alpha}$-independent quadrature method. 
Our implementation is thus given below for future reference, which in spirit follows the procedures introduced by GómezCarrasco and Roncero. ${ }^{26}$

(1) Expressing the product wave function in the reactant BF frame $\phi_{v_{\nu}, j_{\nu}, K_{\alpha}}\left(R_{\nu 0} ; r_{\nu}, \theta_{\nu}\right)$, then calculate product wave function values $\phi_{v_{\nu}, j_{\nu}, K_{\alpha}}\left(r_{i j} ; R_{\alpha i}, \theta_{\alpha j}\right)$ or $\phi_{v_{\nu}, j_{\nu}, K_{\alpha}}\left(R_{i j} ; r_{\alpha i}, \theta_{\alpha j}\right)$ on the reactant coordinate grid.

(2) Construct the transform matrix $U_{i, j, m}$ given by

$$
U_{i, j, m}=g^{r} \frac{2}{\sqrt{\left(r_{e}-r_{b}\right)\left(N_{r}+1\right)}} \sum_{I=1}^{N_{r}} \sin \frac{I \pi\left(r_{i j}-r_{b}\right)}{r_{e}-r_{b}} \sin \frac{m \pi I}{N_{r}+1}
$$

or

$$
\begin{aligned}
U_{i, j, m}= & g R \frac{2}{\sqrt{\left(R_{e}-R_{b}\right)\left(N_{R}+1\right)}} \\
& \times \sum_{I=1}^{N_{R}} \sin \frac{I \pi\left(R_{i j}-R_{b}\right)}{R_{e}-R_{b}} \sin \frac{m \pi I}{N_{R}+1}
\end{aligned}
$$

depending on which pair $\left(R_{\alpha i}, \theta_{\alpha j}\right)$ or $\left(r_{\alpha i}, \theta_{\alpha j}\right)$ is chosen to define the intermediate coordinates.

(3) At each propagation step, the wave packet on the grid points representing the product wave function at the projection plane $R_{\nu 0}$ is obtained by

$$
\begin{aligned}
\psi\left(k, r_{i j} ; R_{\alpha i}, \theta_{\alpha j}, K_{\alpha}\right) \\
=\sum_{m=1}^{N_{r}} \bar{\Psi}\left(k ; R_{\alpha i}, r_{\alpha m}, \theta_{\alpha j}, K_{\alpha}\right) U(i, j, m)
\end{aligned}
$$

or by

$$
\begin{aligned}
\psi\left(k, R_{i j} ; r_{\alpha i}, \theta_{\alpha j}, K_{\alpha}\right) \\
=\sum_{m=1}^{N_{R}} \bar{\Psi}\left(k ; r_{\alpha i}, R_{\alpha m}, \theta_{\alpha j}, K_{\alpha}\right) U(i, j, m),
\end{aligned}
$$

where $\bar{\Psi}$ indicates the wave packet in momentum space of $r_{\alpha}$ in Eq. (30) or of $R_{\alpha}$ in Eq. (31). The $C_{v_{\nu} j_{\nu} K_{\alpha}}(k)$ can be evaluated by calculating the overlap integration

$$
\begin{aligned}
& C_{v_{\nu} j_{\nu} K_{\alpha}}(k) \\
& \quad=\sum_{i, j} \phi_{v_{\nu}, j_{\nu}, K_{\alpha}}\left(r_{i j} ; R_{\alpha i}, \theta_{\alpha j}\right) \psi\left(k, r_{i j} ; R_{\alpha i}, \theta_{\alpha j}, K_{\alpha}\right)
\end{aligned}
$$

or

$$
\begin{aligned}
& C_{v_{\nu} j_{\nu} K_{\alpha}}(k) \\
& \quad=\sum_{i, j} \phi_{v_{\nu}, j_{\nu}, K_{\alpha}}\left(R_{i j} ; r_{\alpha i}, \theta_{\alpha j}\right) \psi\left(k, R_{i j} ; r_{\alpha i}, \theta_{\alpha j}, K_{\alpha}\right) .
\end{aligned}
$$

The $S$-matrix then can be evaluated after a BF transform of $C_{v_{\nu} j_{\nu} K_{\alpha}}(k)$, similar to that in the collocation RCB method. For product channel $\mathrm{A}+\mathrm{BC} \rightarrow \mathrm{AB}+\mathrm{C}, g^{r}$ and $r_{i j}$ in Eq. (28), and $g^{R}$ and $R_{i j}$ in Eq. (29) are given by

$$
g_{i j}^{r} \cdot g_{i j}^{r}=\frac{\mathcal{B}^{2} R_{\beta 0}}{\mathcal{A} \sqrt{R_{\alpha i}^{2}\left(\cos ^{2} \theta_{\alpha j}-1\right)+\left(\mathcal{B} R_{\beta 0}\right)^{2}}} \frac{R_{\beta 0}^{2} r_{\beta}^{2}}{R_{\alpha i}^{2} r_{i j}^{2}},
$$

$$
r_{i j}=\frac{1}{\mathcal{A}}\left[R_{\alpha i} \cos \theta_{\alpha j}+\sqrt{R_{\alpha i}^{2}\left(\cos ^{2} \theta_{\alpha j}-1\right)+\left(\mathcal{B} R_{\beta 0}\right)^{2}}\right],
$$

$$
\begin{aligned}
& g_{i j}^{R} \cdot g_{i j}^{R}=\frac{\mathcal{B}^{2} R_{\beta 0}}{\sqrt{\left(\mathcal{A} r_{\alpha i}\right)^{2}\left(\cos ^{2} \theta_{\alpha j}-1\right)+\left(\mathcal{B} R_{\beta 0}\right)^{2}}} \frac{R_{\beta 0}^{2} r_{\beta}^{2}}{R_{i j}^{2} r_{\alpha i}^{2}}, \\
& R_{i j}=\mathcal{A} r_{\alpha i} \cos \theta_{\alpha j}+\sqrt{\left(\mathcal{A} r_{\alpha i}\right)^{2}\left(\cos ^{2} \theta_{\alpha j}-1\right)+\left(\mathcal{B} R_{\beta 0}\right)^{2}},
\end{aligned}
$$

where $\mathcal{A}=\left(M_{A}+M_{B}\right) / M_{A}-M_{C} /\left(M_{B}+M_{C}\right)$ and $\mathcal{B}=\left(M_{A}\right.$ $\left.+M_{B}\right) / M_{A}$.

For product channel $\mathrm{A}+\mathrm{BC} \rightarrow \mathrm{AC}+\mathrm{B}$, the same equations can be used to calculate $g^{r}$ and $r_{i j}$ in Eq. (28), and $g^{R}$ and $R_{i j}$ in Eq. (29), but with $\mathcal{A}=\left(M_{A}+M_{C}\right) / M_{A}-M_{B} /\left(M_{B}\right.$ $\left.+M_{C}\right), \mathcal{B}=\left(M_{A}+M_{C}\right) / M_{A}$, and set $\theta_{\alpha j}$ as $\pi-\theta_{\alpha j}$. We note that there are typos in Eq. (A4) and Eq. (A5) in Ref. 26, which are for the other product channel, instead of the channel specified in the main content.

\section{RESULTS AND DISCUSSION}

In this section, we illustrate the applicability of the two RCB methods in several numerical examples. We start by demonstrating the utility of the RCB methods in calculating the DCS for the $\mathrm{H}+\mathrm{HD}$ reaction. This is followed by an examination of the $\mathrm{Cl}+\mathrm{H}_{2}$ reaction. The van der Waals well in the $\mathrm{Cl}+\mathrm{H}_{2}$ reactant channel makes this system an ideal case to test the efficiency provided by a $L$-shaped grid in state-to-state calculations. We then examine the effects of the projection position using the $\mathrm{H}+\mathrm{HCl}$ reaction as an example. Finally, we discuss the relative merits of $\mathrm{PCB}$ and $\mathrm{RCB}$ methods.

\section{A. Demonstration of methods: $\mathrm{H}+\mathrm{HD}$ reaction}

The $\mathrm{H}+\mathrm{H}_{2}$ exchange reaction and its isotopic variants have been extensively studied over many years. ${ }^{53}$ It is also a benchmark reaction for the development of new theoretical methods. Aoiz et al. ${ }^{54}$ recently gave a comprehensive progress review on the dynamical study of this reaction. An excellent agreement between theory and experiment has been achieved for this reaction on the BKMP2 potential energy surface (PES) ${ }^{55-58}$ as well as on the latest CCI PES. ${ }^{59,60}$ Here, the BKMP2 PES is employed for the $\mathrm{H}+\mathrm{HD}$ system. It is noted that our calculations reported here did not account for the Fermi-Dirac statistics. ${ }^{61,62,57}$

The parameters used in the calculations on a $L$-shaped grid are listed in Table I. The usage of the $L$-shaped grid for this reaction does not save much grid points, comparing with a calculation with a rectangular-shape grid, because there is no long range interaction potential in this system.

Product rotational state-resolved DCSs at collision energies of 0.4 and $0.8 \mathrm{eV}$ for $\mathrm{H}+\mathrm{H}^{\prime} \mathrm{D}\left(v_{0}=0, j_{0}=1\right) \rightarrow \mathrm{HH}^{\prime}\left(v^{\prime}\right.$ $\left.=0, j^{\prime}=0,5\right)+\mathrm{D} \quad\left(\right.$ left panels) $\mathrm{H}+\mathrm{H}^{\prime} \mathrm{D}\left(v_{0}=0, j_{0}=1\right)$ $\rightarrow \mathrm{HD}\left(v^{\prime}=0, j^{\prime}=0,5\right)+\mathrm{H}^{\prime}$ (right panels), calculated by both coordinate transform RCB and collocation RCB methods are shown in Fig. 1. The agreement between the two is excellent. Since we have shown earlier that the results from the bench- 
TABLE I. Parameters used in the numerical calculations. (Atomic units are used if not otherwise stated.)

\begin{tabular}{|c|c|c|c|}
\hline & $\mathrm{H}+\mathrm{HD}$ & $\mathrm{H}_{2}+\mathrm{Cl}$ & $\mathrm{H}+\mathrm{HCl}$ \\
\hline Grid/basis range and size & $\begin{array}{l}R \in[0.1,12.0], N_{R}^{1}=63, N_{R}^{2}=49 \\
r \in[0.4,9.0], N_{r}^{1}=49, N_{r}^{2}=23 \\
j_{\min }=0 \sim j_{\max }=46, N_{j}=47\end{array}$ & $\begin{array}{l}R \in[0.1,23.0], N_{R}^{1}=179, N_{R}^{2}=79 \\
r \in[0.6,16.0], N_{r}^{1}=89, N_{r}^{2}=23 \\
j_{\min }=0 \sim j_{\max }=80, N_{j}=41\end{array}$ & $\begin{array}{l}R \in[0.8,16.0], N_{R}^{1}=129, N_{R}^{2}=127 \\
r \in[1.2,16.0], N_{r}^{1}=127, N_{r}^{2}=29 \\
j_{\min }=0 \sim j_{\max }=140, N_{j}=141\end{array}$ \\
\hline Initial wave packet & $R_{c}=8.0$ & $R_{c}=8.0,10.0,12.0,14.0,16.0,18.0$ & $R_{c}=11.0$ \\
\hline $\exp \left[-\left(R-R_{c}\right)^{2} /\left(2 \Delta_{R}^{2}\right)\right] \cos \left(k_{0} R\right)$ & $\begin{array}{l}\Delta_{R}=0.5 \\
k_{0}=\sqrt{2 E_{0} \mu_{R}} \text { with } E_{0}=0.7 \mathrm{eV}\end{array}$ & $\begin{array}{l}\Delta_{R}=0.25 \\
k_{0}=\sqrt{2 E_{0} \mu_{R}} \text { with } E_{0}=0.45 \mathrm{eV}\end{array}$ & $\begin{array}{l}\Delta_{R}=0.16 \\
k_{0}=\sqrt{2 E_{0} \mu_{R}} \text { with } E_{0}=1.7 \mathrm{eV}\end{array}$ \\
\hline Absorbing potential & $\begin{array}{l}C^{\prime}=0.1, n^{\prime}=n=2, R_{a}=9.5 \\
C_{a}=0.12, C_{b}=0.3, r_{a}=6.7, r_{b}=8.9\end{array}$ & $\begin{array}{l}C^{\prime}=0.03, n^{\prime}=n=2, R_{a}=19.0 \\
C_{a}=0.01, C_{b}=0.2, r_{a}=12.0, r_{b}=13.5\end{array}$ & $\begin{array}{l}C^{\prime}=0.07, n^{\prime}=n=2, R_{a}=12.0 \\
C_{a}=0.05, C_{b}=0.2, r_{a}=12.0, r_{b}=13.0\end{array}$ \\
\hline Total propagation time & 1200 iterations & 2000 iterations & 2000 iterations \\
\hline Matching plane & $R_{0}^{\prime}=5.5$ & $R_{0}^{\prime}=8.0$ & $R_{0}^{\prime A}=8.0,9.0,10.0, R_{0}^{\prime E}=9.0,10.0,11.0$ \\
\hline
\end{tabular}

mark time-independent method (the $\mathrm{ABC}$ code) agree very well with those from the collocation RCB method, we can claim that both the RCB methods are capable of extracting accurate state-to-state information in both product channels simultaneously. The coordinate transform RCB method does not require extra grid points.

\section{B. Advantages of $L$-shaped grid: $\mathrm{Cl}+\mathrm{H}_{2}$ reaction}

An extensive body of work, both theoretical ${ }^{63-68}$ and experimental, ${ }^{69-74}$ also exists for the $\mathrm{Cl}+\mathrm{H}_{2}$ system and its isotopic variations. This system has played a central role in chemical kinetics and has served as a test case for bimolecular reaction rate theory. ${ }^{75,76}$ The adiabatic BW PES (Ref. 68) reported in 2000 yielded quantum thermal rate constants which agree perfectly with experiment over a wide range of temperatures. ${ }^{77,66}$ However, the recent focus has been on the disagreement between theory and experiment for the $\mathrm{Cl}\left({ }^{2} P \frac{1}{2} / \frac{3}{2}\right)+\mathrm{H}_{2}$ reaction including spin-orbit effects. ${ }^{64,72,78-81}$ In the following numerical calculations, the benchmark BW PES $^{68}$ is used.
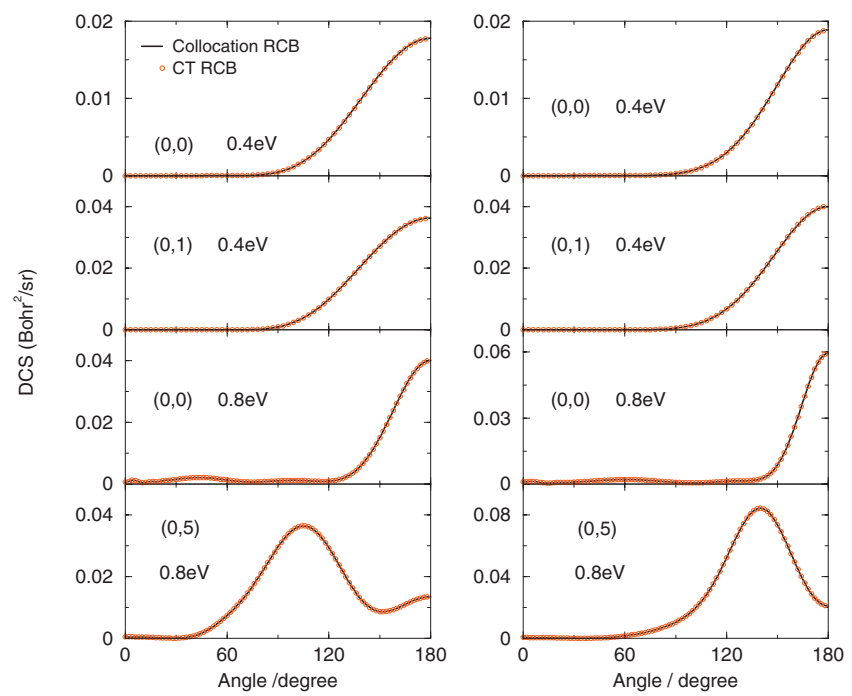

FIG. 1. State-to-state DCSs at collision energies of 0.4 and $0.8 \mathrm{eV}$ of the $\mathrm{H}+\mathrm{H}^{\prime} \mathrm{D}\left(v_{0}=0, j_{0}=1\right) \rightarrow \mathrm{H}^{\prime}+\mathrm{HD}\left(v^{\prime}=0, j^{\prime}=0\right.$ and 5) reaction (left column) and of the $\mathrm{H}+\mathrm{H}^{\prime} \mathrm{D}\left(v_{0}=0, j_{0}=1\right) \rightarrow \mathrm{D}+\mathrm{HH}^{\prime}\left(v^{\prime}=0, j^{\prime}=0\right.$ and 5) reaction (right column), calculated using the collocation and coordinate transform RCB methods. It is seen that the two methods give identical and converged DCSs for this reaction with parameters listed in Table I.
The numerical parameters are given in Table I, and the resulting two-dimensional (2D) PES of $R$ and $r$ with minimization along $\theta$ is given in Fig. 2. The maximum grid range of $R$ has to be large because the van der Waals well in the reactant $\mathrm{Cl}+\mathrm{H}_{2}$ channel has an important effect on the total reaction probability. ${ }^{82}$ As shown in Fig. 3, different starting positions of the initial wave packet lead to quite different total reaction probabilities. It is clearly seen from Fig. 2 that the $L$-shaped gird saves substantial computational costs, facilitated by the small skew angle of the product channel. The total reaction probabilities obtained by both the flux method and state-to-state method agree with each other very well and our results indicate $K_{\max }=9$ is sufficient to converge the DCS up to collision energy of $1.0 \mathrm{eV}$. Because this reaction has been calculated many times quantum mechanically, the DCSs are not presented here for brevity.

\section{Position of projection plane: $\mathrm{H}+\mathrm{HCl}$ reaction}

Despite a large number of kinetic studies on the $\mathrm{H}$ $+\mathrm{HCl}$ reaction in the temperature range of $195 \mathrm{~K} \leq T$ $\leq 1200 \mathrm{~K},{ }^{83-86}$ there are only a few experimental and theoretical studies on the reaction dynamics. ${ }^{87-93}$ Branchett et al. ${ }^{94}$ recently reported the $\mathrm{H}+\mathrm{HCl} \rightarrow \mathrm{H}_{2}+\mathrm{Cl}$ reactive cross

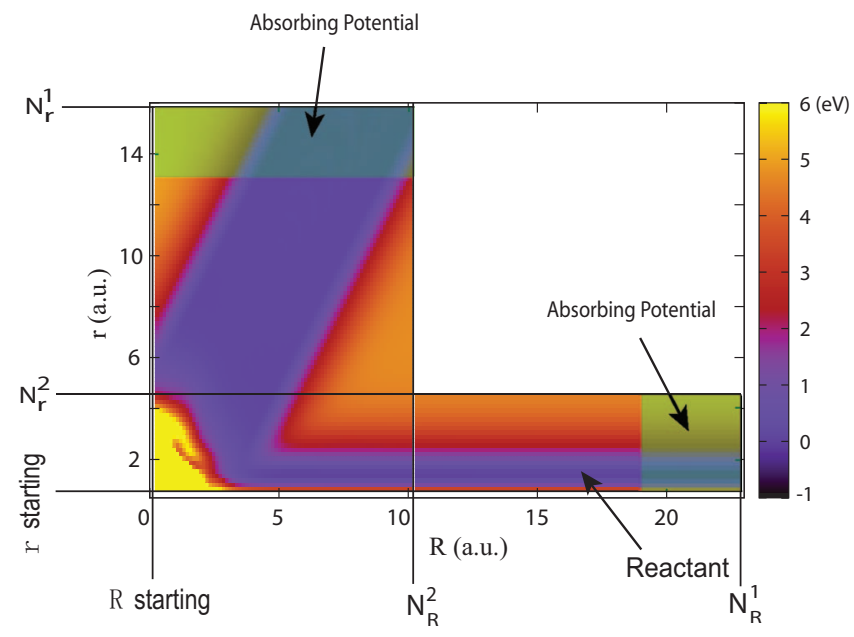

FIG. 2. 2D contours of the potential energy surface of the $\mathrm{Cl}+\mathrm{H}_{2}$ reaction along $R$ and $r$ degrees of freedom on the $L$-shaped grid with the energy in the angular coordinate optimized. The parameters in Table I are defined in the $2 \mathrm{D}$ plot. 


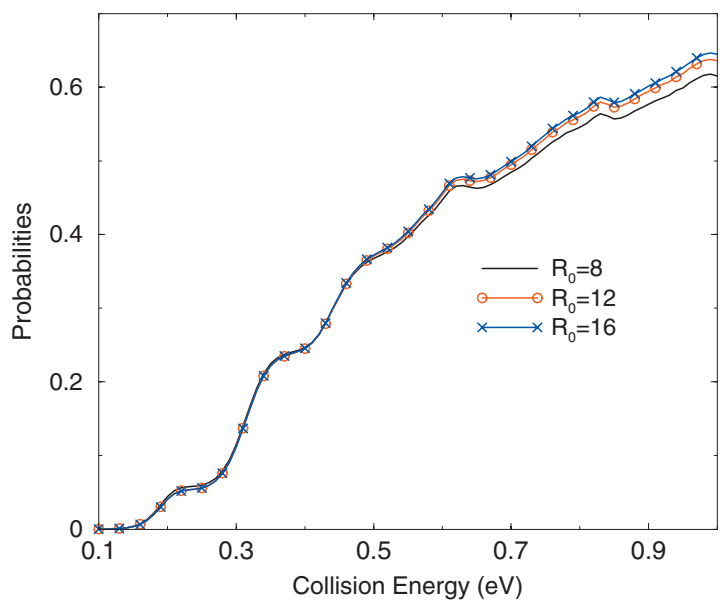

FIG. 3. Total reaction probabilities obtained from initial $\mathrm{Cl}+\mathrm{H}_{2}$ wave packet placed at different positions $R_{c}=8.0,12.0$, and 16.0 a.u. The results indicate that the entire van der Waals well in the reactant channel should be included for an accurate calculation of the total reaction probabilities above the collision energy of $0.45 \mathrm{eV}$.

sections at a state-to-state level using time-independent quantum method on the GQQ PES. ${ }^{95}$ Yao et al. ${ }^{96}$ studied the total reaction probabilities of the $\mathrm{H}+\mathrm{HCl}$ reactive scattering with a TDWP method using the BW PES. ${ }^{68}$ (Noted that there are errors in the work of Yao et al. ${ }^{96}$ ) In the following, the state-to-state cross sections of the abstraction reaction $\mathrm{H}$ $+\mathrm{HCl} \rightarrow \mathrm{H}_{2}+\mathrm{Cl}$ and the exchange reaction $\mathrm{H}+\mathrm{H}^{\prime} \mathrm{Cl} \rightarrow \mathrm{HCl}$ $+\mathrm{H}^{\prime}$ are reported for the first time on the BW PES, using the coordinate transform RCB method. As noted earlier, the cross sections of both product channels were calculated at the same time with a single wave packet propagated in reactant Jacobi coordinates.

We have seen in the previous subsection that because of the long-range interaction potential in the $\mathrm{Cl}+\mathrm{H}_{2}$ channel, the $L$-shaped grid leads to significant savings for the $\mathrm{Cl}$ $+\mathrm{H}_{2}$ reaction. It would be interesting to see if the same van der Waals well requires a large $r$ grid for calculating product states-resolved results for the $\mathrm{Cl}+\mathrm{H}_{2}$ and $\mathrm{H}+\mathrm{HCl}$ products.

The parameters used in the calculation are listed in Table I. $K_{\max }=19$ was adopted for the results shown below, whose convergence was assured by a calculation using $K_{\max }=24$. It turns out in this case that the $L$-shape grid does not save much points, as compared with a rectangular grid. This is because the large skew angle of the abstraction reaction (channel A) $\mathrm{H}+\mathrm{HCl} \rightarrow \mathrm{Cl}+\mathrm{H}_{2}$. In Fig. 4, the total reaction probabilities of channels $\mathrm{A}$ and $\mathrm{E}$ (the exchange reaction $\mathrm{H}$ $\left.+\mathrm{H}^{\prime} \mathrm{Cl} \rightarrow \mathrm{H}^{\prime}+\mathrm{HCl}\right)$ at different total angular momentum $J$ values are shown.

We will now focus here on positions of the projection planes and their effects on the final results. It is often believed that the product state-resolved information has to be extracted at a projection plane far into the asymptotic region, ${ }^{26,21}$ which may lead to huge grid range requirement for a state-to-state calculation. To examine this issue, the total reaction probabilities calculated by both the flux and state-to-state methods are compared in Fig. 5 for several $J$ values. The positions of the projection planes $\left(R_{0}^{A}, R_{0}^{E}\right)$ are $(8.0,9.0),(9.0,10.0)$ and $(10.0,11.0)$ a.u., respectively. In Figs. 6 and 7, the product state-resolved ICSs calculated at

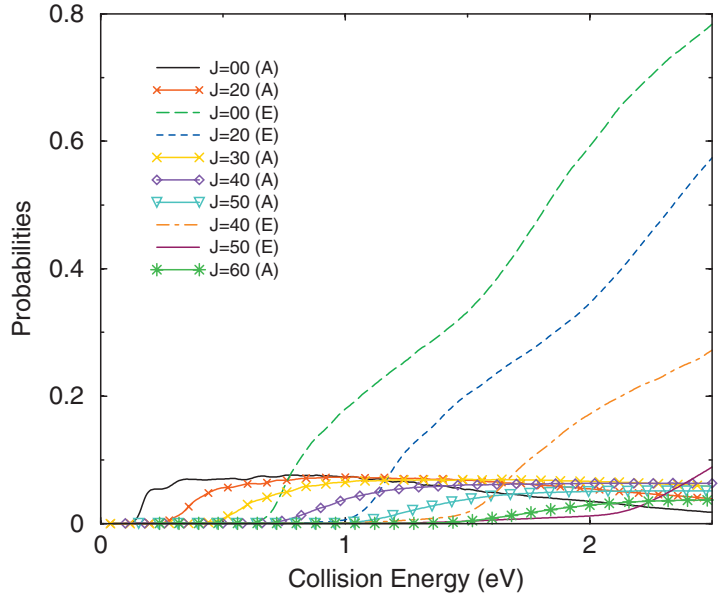

FIG. 4. Total reaction probabilities for the $\mathrm{H}+\mathrm{HCl}$ reaction obtained by the state-to-state reaction probabilities for $J=0,20,30,40,50$, and 60 of the abstraction reaction (a) and exchange reaction (e).

different $R_{0}^{A}$ and $R_{0}^{E}$ indicates that there are negligible differences between them, even when the projection planes are far from the true asymptotic region. Of course the convergence of ICS does not necessarily guarantee the convergence of DCS, because phases of $S$-matrix elements may depend sensitively on $R_{0}^{A}$ or $R_{0}^{E}$. However, as seen in Figs. 8 and 9, the total and rovibrationally resolved DCSs extracted at different projection planes are mostly identical by visual inspection. These results suggest that the van der Waals well in the $\mathrm{Cl}$ $+\mathrm{H}_{2}$ product channel has little effect on the product stateresolved reaction probabilities, presumably due to the weak coupling between the translational and internal degrees of freedom.
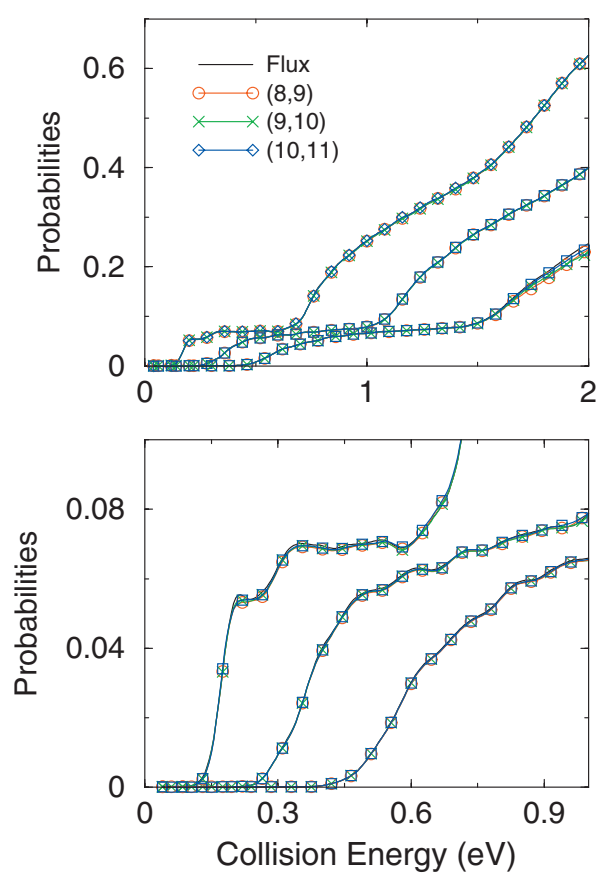

FIG. 5. Comparison between the total reaction probabilities for the $\mathrm{H}$ $+\mathrm{HCl}$ reaction obtained by summing the state-to-state reaction probabilities with those calculated directly by a flux method for $J=0,20$, and 40 . The upper panel is for the total $(\mathrm{A}+\mathrm{E})$ reaction while the lower panel is for the abstraction reaction only. 

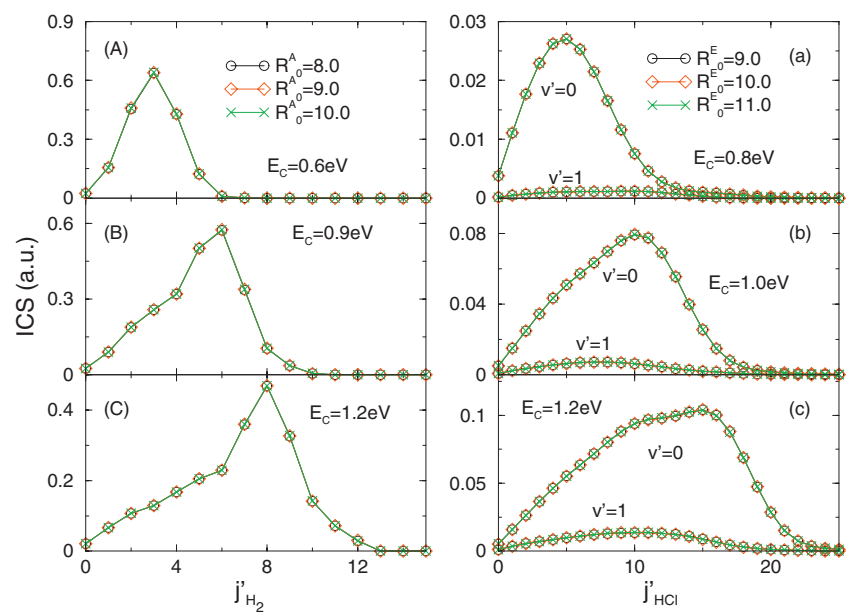

FIG. 6. A comparison for a state-to-state ICSs for the $\mathrm{H}+\mathrm{HCl}$ reaction, at selected collision energies of $0.4,0.8$, and $1.2 \mathrm{eV}$ for the abstraction reaction (left panels) and $0.8,1.0$, and $1.2 \mathrm{eV}$ for the exchange reaction (right panel). The product analysis planes are placed at $R_{0}^{A}=8.0,9.0$, and 10.0 a.u. for the abstraction reaction and at $R_{0}^{B}=9.0,10.0$, and 11.0 a.u. for the exchange reaction. The positions of the product state analysis plane has little influence on the results, even they are not at the true asymptote.

These observations seem to suggest that the projection plane may not need to be placed in the true asymptotic region, if the final state interaction is small. This conclusion favors the RCB approach because an unreasonably large grid is not needed. In the $\mathrm{H}+\mathrm{HCl}$ case, the projection planes placed at $(8.0,9.0)$ a.u. allow a smaller $r$ grid and accordingly a smaller spherical basis as well. For systems with strong asymptotic interactions, which we believe to be small in number, one can always check the convergence of the results by pushing the projection plane farther into the asymptote, as suggested by step (4) in our original proposal. ${ }^{20}$

It should be pointed that the different requirements for the position of the initial wave packet for $\mathrm{H}_{2}+\mathrm{Cl}$ reaction and the position of the product state analysis plane for $\mathrm{H}$ $+\mathrm{HCl}$ reaction is likely due to the fact that the initial wave packet for the $\mathrm{H}_{2}+\mathrm{Cl}$ reaction has the lowest rovibrational state. On the other hand, there are many excited internal states for the $\mathrm{H}+\mathrm{HCl}$ reaction, which are less sensitive to the potential energy surface. This is borne out by the numerical
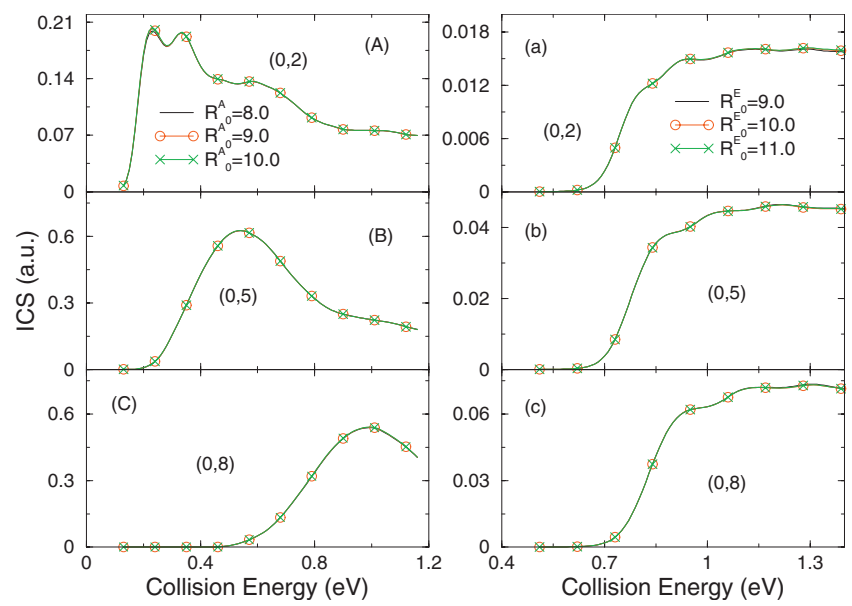

FIG. 7. Same as in Fig. 6, but for a comparison for a state-to-state ICSs as a function of collision energy.

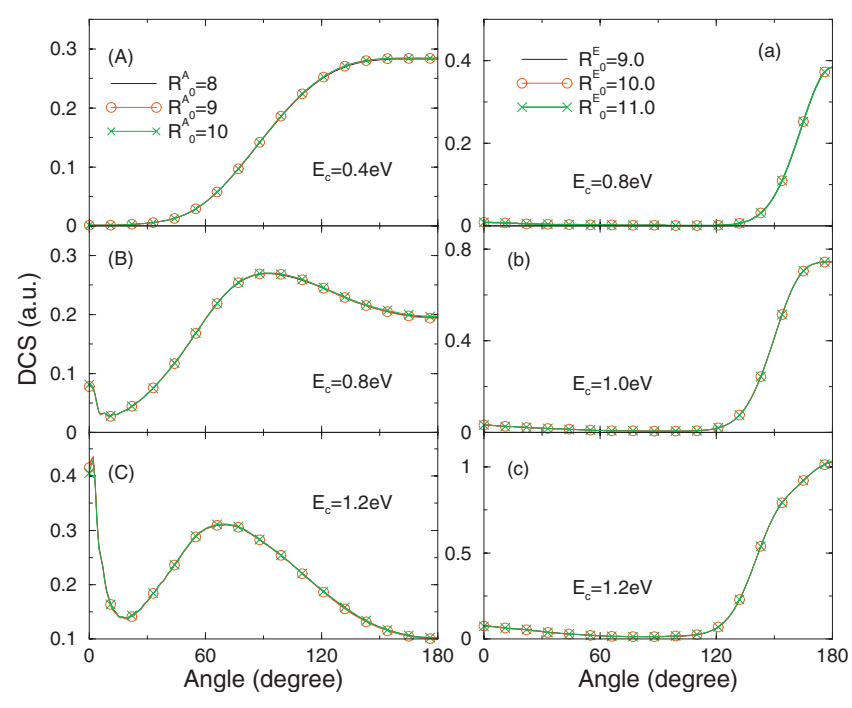

FIG. 8. Same as in Fig. 7, but for a comparison of the total DCSs.

results in Fig. 9. We also note in passing that the state-tostate results reported here indicate that the abstraction reaction and exchange reaction of $\mathrm{H}+\mathrm{H}^{\prime} \mathrm{Cl}$ are essentially similar to those of $\mathrm{H}+\mathrm{HBr}$ reaction. ${ }^{38}$

\section{Choice between the PCB and RCB methods}

In the work of Gómez-Carrasco and Roncero, ${ }^{26}$ a detailed discussion was given on how to choose between the PCB and RCB methods, with particular relevance to $a$ and $c$ in Eqs. (26) and (27) and the number of the necessary $K$ blocks needed to converge state-to-state scattering attributes. For $J=0$, reactant coordinates are more efficient for $a \gg c$ while product coordinates are preferred otherwise. For $J$ $>0$, product coordinates are more computationally efficient if $\mathcal{A} \approx 0$ because the helicity number required in a $\mathrm{PCB}$ method is proportional to $\mathcal{A}$. Their conclusions were reached by using a rectangular grid. Giving the fact that the PCB method cannot usually take full advantages of the $L$-shaped grid because the grid range in a PCB method is mostly determined by the expansion of the initial wave packet in product coordinates, we can expect that with the adoption of the
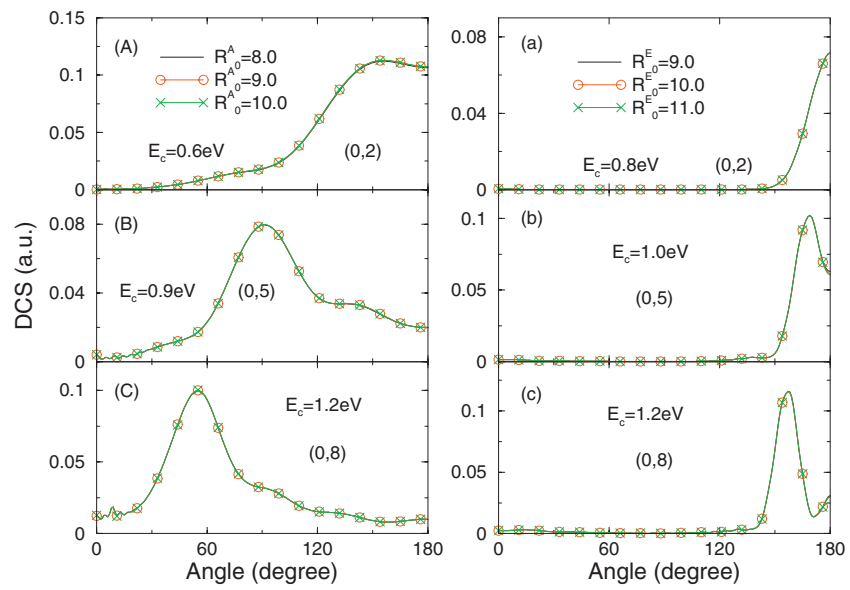

FIG. 9. Same as in Fig. 8, but for a comparison at a state-to-state level for product states as $\left(v^{\prime}, j^{\prime}\right)=(0,2),(0,5)$ and $(0,8)$. 
$L$-shaped grid, the RCB method would in most cases have overwhelming advantages over the PCB method.

The use of $a$ and $c$ in choosing between the PCB and $\mathrm{RCB}$ methods has a clear physical origin. In a triatomic wave packet calculation, the transformation between the basis and grid representations for $r$ and $R$ is achieved by fast Fourier transform. Therefore, the basis size for the angular degree of freedom is the computational bottleneck, especially with multiple helicity blocks. Note that $a * M_{B}\left(b * M_{B}\right)$ is the reduced mass for the diatom $\mathrm{BC}(\mathrm{AB})$, which is proportional to the moment of inertia. In a typical situation, the larger the moment of inertia, the larger the angular basis is needed. Of course, this criterium needs be used in combination with the exothermicity of the reaction, which might tip the balance between the two.

$\mathrm{The} \mathrm{HCl}+\mathrm{H}$ reaction offers a good example for illustrating the choices. The reaction is nearly thermal neutral. Therefore if only the abstraction reaction $\mathrm{H}+\mathrm{HCl} \rightarrow \mathrm{H}_{2}+\mathrm{Cl}$ is concerned, the PCB method may be a better choice, especially for $J=0$. However, if only the exchange reaction $\mathrm{H}$ $+\mathrm{H}^{\prime} \mathrm{Cl} \rightarrow \mathrm{HCl}+\mathrm{H}^{\prime}$ is of interest, the RCB method is much better because the PCB method requires much more helicity components. Since the state-resolved information of the two product channels can be calculated at the same time using the RCB method, it is clear that the RCB method is more computationally suitable for this reaction.

Finally, we should comment on the effect of exchange symmetry. Let us use the $\mathrm{H}+\mathrm{HCl} \rightarrow \mathrm{H}_{2}+\mathrm{Cl}$ reaction again as an example. The use of PCB in this case allows one to take advantage of the exchange symmetry in the $\mathrm{H}_{2}$ reactant, while such symmetry is difficult to realize in the reactant coordinates. On the other hand, the RCB approach is obviously preferred as it offers the advantages of both the $L$-shaped grid and symmetry adaptation.

This conclusion is drawn based on our method, i.e., a $K_{\alpha}$-dependent angular quadrature is adopted and a uniform $L$-shape grid points are applied for all angular points. When different $L$-shape grids used for different angular points, it might lead to a more efficient procedure. In some cases, the $L$-shape grid method can results in a more grid point savings in a PCB method than in a RCB method. Further investigation is required before we reach a definite conclusion.

\section{CONCLUSIONS}

Two RCB wave packet methods for extracting state-tostate reactive scattering information in a triatomic system are reviewed and compared. It is concluded that both methods can be efficiently used to extract the product states-resolved attributes in both product channels $\mathrm{A}+\mathrm{BC} \rightarrow \mathrm{AB}+\mathrm{C}$ and $\mathrm{AC}+\mathrm{B}$. The coordinate transform $\mathrm{RCB}$ method, introduced by Gómez-Carrasco and Roncero, ${ }^{26}$ is in general more efficient than the collocation RCB method introduced by Sun et $a l .{ }^{20}$ Neither method requires a denser reactant grid than that in the traditional wave packet method for total reaction probabilities calculation using a flux method.

The adoption of a $L$-shaped grid in a RCB method saves considerable grid points for many reactions, especially for those whose long range interaction potential in the reactant channel is important and the product channel has a small skew angle. We conclude that RCB methods should be computationally more effective than the PCB method for most triatomic reactive reaction systems at the state-to-state level.

\section{ACKNOWLEDGMENTS}

Z.G.S. and D.H.Z. were supported by the Knowledge Innovation Program of the Chinese Academy of Science (DICP Grant Nos. R200402 and Y200601) and by the National Natural Science Foundation of China (Grant Nos. 20688301 and 20773127). H.G. was supported by the U.S. Department of Energy (Grant No. DE-FG02-05ER15694).

${ }^{1}$ G. C. Schatz and A. Kuppermann, J. Chem. Phys. 65, 4642 (1976).

${ }^{2}$ S. C. Althorpe and D. C. Clary, Annu. Rev. Phys. Chem. 54, 493 (2003). ${ }^{3}$ D. Skouteris, J. F. Castillo, and D. E. Manolopoulos, J. Comput. Phys. 133, 128 (2000).

${ }^{4}$ V. Aquilanti, S. Cavalli, D. De Fazio, A. Volpi, X. Giménez, and J. M. Lucas, Phys. Chem. Chem. Phys. 4, 401 (2002).

${ }^{5}$ P. Honvault and J.-M. Launay, J. Chem. Phys. 111, 6665 (1999).

${ }^{6}$ P. Honvault and J.-M. Launay, J. Chem. Phys. 114, 1057 (2001).

${ }^{7}$ N. Balucani, P. Casavecchia, L. Bañares, F. J. Aoiz, T. Gonzalez-Lezana, P. Honvault, and J.-M. Launay, J. Phys. Chem. A 110, 817 (2006).

${ }^{8}$ L. Banares, J. F. Castillo, P. Honvault, and J.-M. Launay, Phys. Chem. Chem. Phys. 7, 627 (2005)

${ }^{9}$ N. Balucani, G. Capozza, L. Cartechini, A. Bergeat, R. Bobbenkamp, P. Casavecchia, F. J. Aoiz, L. Banares, P. Honvault, B. Bussery-Honvault, and J.-M. Launay, Phys. Chem. Chem. Phys. 6, 4957 (2004).

${ }^{10}$ P. Honvault, S. Y. Lin, D. Q. Xie, and H. Guo, J. Phys. Chem. A 111, 5349 (2007).

${ }^{11}$ D. H. Zhang and J. Z. H. Zhang, J. Chem. Phys. 101, 1146 (1994).

${ }^{12}$ D. H. Zhang and J. C. Light, J. Chem. Phys. 104, 4544 (1996).

${ }^{13}$ M. H. Yang, S.-Y. Lee, and D. H. Zhang, J. Chem. Phys. 126, 064303 (2007).

${ }^{14}$ F. Gögtas, G. G. Balint-Kurti, and A. R. Offer, J. Chem. Phys. 104, 7927 (1996).

${ }^{15}$ S. Y. Lin and H. Guo, Phys. Rev. A 74, 022703 (2006).

${ }^{16}$ K. J. Yuan, Y. Cheng, X. H. Liu, S. Harich, and X. M. Yang, Phys. Rev. Lett. 96, 103202 (2006).

${ }^{17}$ S. C. Althorpe and T. Seideman, J. Chem. Phys. 113, 7901 (2000).

${ }^{18}$ T. Peng and J. Z. H. Zhang, J. Chem. Phys. 105, 6072 (1996).

${ }^{19}$ T. Peng, W. Zhu, D. Y. Wang, and J. Z. H. Zhang, Faraday Discuss. 110, 159 (1998).

${ }^{20}$ Z. Sun, X. Xu, S. Y. Lee, and D. H. Zhang, J. Phys. Chem. A 113, 4145 (2009).

${ }^{21}$ Z. Sun, D. H. Zhang, C. Xu, S. Zhou, D. Xie, G. Lendvay, S. Y. Lee, S. Y. Lin, and H. Guo, J. Am. Chem. Soc. 130, 14962 (2008).

${ }^{22}$ Z. Sun, S. Y. Lee, H. Guo, and D. H. Zhang, J. Chem. Phys. 130, 174102 (2009).

${ }^{23}$ Z. Sun, S. Y. Lee, H. Guo, and D. H. Zhang, J. Chem. Phys. 131, 049906 (2009).

${ }^{24}$ Z. Li, D. Q. Xie, Z. Sun, D. H. Zhang, S. Y. Lin, and H. Guo, J. Chem. Phys. 131, 124313 (2009).

${ }^{25}$ Z. Sun, L. Liu, S. Y. Lin, R. Schinke, H. Guo, and D. H. Zhang, Proc. Natl. Acad. Sci. U.S.A. 107, 555 (2010).

${ }^{26}$ S. Gómez-Carrasco and O. Roncero, J. Chem. Phys. 125, 054102 (2006).

${ }^{27}$ A. Zanchet, O. Roncero, T. González-Lezana, A. Rodríguez-Lopéz, A. Aguado, C. Sanz-Sanz, and S. Gómez-Carrasco, J. Phys. Chem. A 113, 14488 (2009).

${ }^{28}$ R. Chen and H. Guo, Comput. Phys. Commun. 119, 19 (1999).

${ }^{29}$ E. M. Goldfield and S. K. Gray, Adv. Chem. Phys. 136, 1 (2007).

${ }^{30}$ G. G. Balint-Kurti, Int. Rev. Phys. Chem. 27, 507 (2008).

${ }^{31}$ R. T. Pack and G. A. Packer, J. Chem. Phys. 87, 3888 (1987).

${ }^{32}$ S. K. Gray and G. G. Balint-Kurti, J. Chem. Phys. 108, 950 (1998).

${ }^{33}$ M. Hankel, S. C. Smith, S. K. Gray, and G. G. Balint-Kurti, Comput. Phys. Commun. 179, 569 (2008).

${ }^{34}$ J. C. Light and T. Carrington, Jr., Adv. Chem. Phys. 114, 263 (2000).

${ }^{35}$ D. H. Zhang and J. Z. H. Zhang, J. Chem. Phys. 99, 5615 (1993).

${ }^{36}$ D. H. Zhang and J. Z. H. Zhang, J. Chem. Phys. 100, 2697 (1994).

${ }^{37}$ Z. T. Cai, D. H. Zhang and J. Z. H. Zhang, J. Chem. Phys. 100, 5631 
(1994).

${ }^{38}$ B. Fu and D. H. Zhang, J. Phys. Chem. A 111, 9516 (2007).

${ }^{39}$ D. M. Brink and G. R. Satchler, Angular Momentum, 2nd ed. (Clarendon, Oxford, 1968).

${ }^{40}$ S. C. Althorpe, J. Chem. Phys. 114, 1601 (2001).

${ }^{41}$ J. Z. H. Zhang, J. Chem. Phys. 94, 6047 (1991).

${ }^{42}$ R. N. Zare, Angular Momentum (Wiley, New York, 1988).

${ }^{43}$ J. Z. H. Zhang and W. H. Miller, J. Chem. Phys. 91, 1528 (1989).

${ }^{44}$ E. J. Rackham, T. Gonzalez-Lezana, and D. E. Manolopoulos, J. Chem. Phys. 119, 12895 (2003)

${ }^{45}$ V. A. Mandelshtam and H. S. Taylor, J. Chem. Phys. 102, 7390 (1995).

${ }^{46}$ V. A. Mandelshtam and H. S. Taylor, J. Chem. Phys. 103, 2903 (1995).

${ }^{47}$ R. C. Mowrey, J. Chem. Phys. 94, 7098 (1991).

${ }^{48}$ D. H. Zhang and J. Z. H. Zhang, J. Chem. Phys. 101, 3671 (1994).

${ }^{49}$ M. Lara, A. Aguado, M. Paniagua, and O. Roncero, J. Chem. Phys. 113, 1781 (2000).

${ }^{50}$ J. C. Light, I. P. Hamilton, and J. V. Lill, J. Chem. Phys. 82, 1400 (1985).

${ }^{51}$ W. T. Yang and A. C. Peet, Chem. Phys. Lett. 153, 98 (1988).

${ }_{53}^{52}$ J. Echave and D. C. Clary, Chem. Phys. Lett. 190, 225 (1992).

${ }^{53}$ W. H. Miller, Annu. Rev. Phys. Chem. 41, 245 (1990).

${ }^{54}$ F. J. Aoiz, L. Bañares, and V. J. Herrero, Int. Rev. Phys. Chem. 24, 119 (2005).

${ }^{55}$ L. Bañares, F. J. Aoiz, V. J. Herrero, M. J. D’Mello, B. Niederjohann, K. Seekamp-Rahn, E. Wrede, and L. Schnieder, J. Chem. Phys. 108, 6160 (1998).

${ }^{56}$ A. I. Boothroyd, W. J. Keogh, P. G. Martin, and M. R. Peterson, J. Chem. Phys. 104, 7139 (1996).

${ }^{57}$ S. A. Harich, D. X. Dai, X. M. Yang, S. D. Chao, and R. T. Skodje, Nature (London) 419, 281 (2002).

${ }^{58}$ D. X. Dai, C. C. Wang, S. A. Harich, X. Y. Wang, X. M. Yang, S. D. Chao, and R. T. Skodje, Science 300, 1730 (2003).

${ }^{59}$ S. L. Mielke, B. C. Garrett, and K. A. Peterson, J. Chem. Phys. 116, 4142 (2002).

${ }^{60}$ S. L. Mielke, K. A. Peterson, D. W. Schwenke, B. C. Garret, D. G. Truhlar, J. V. Michael, M.-C. Su, and J. W. Sutherland, Phys. Rev. Lett. 91, 063201 (2003)

${ }^{61}$ S. D. Chao, S. A. Harich, D. X. Dai, C. C. Wang, X. M. Yang, and R. T. Skodje, J. Chem. Phys. 117, 8341 (2002).

${ }^{62}$ S. A. Harich, D. X. Dai, X. M. Yang, S. D. Chao, and R. T. Skodje, J. Chem. Phys. 116, 4769 (2002)

${ }^{63}$ J.-B. Song and E. A. Gislason, Chem. Phys. 237, 159 (1998).

${ }^{64}$ M. H. Alexander, G. Capecchi, and H. J. Werner, Science 296, 715 (2002).

${ }^{65}$ M. H. Alexander, G. Capecchi, and H.-J. Werner, Faraday Discuss. 127, 59 (2004)

${ }^{66}$ U. Manthe, G. Capecchi, and H.-J. Werner, Phys. Chem. Chem. Phys. 6, 5026 (2004).

${ }^{67}$ D. Manolopoulos and M. H. Alexander, Phys. Chem. Chem. Phys. 6, 4984 (2004)

${ }^{68}$ W. Bian and H.-J. Werner, J. Chem. Phys. 112, 220 (2000).

${ }^{69}$ D. M. Neumark, A. M. Wodtke, G. N. Robinson, C. C. Hayden, and Y. T.
Lee, Phys. Rev. Lett. 53, 226 (1984).

${ }^{70}$ A. Osterwalder, J. B. Nee, J. Zhou, and D. M. Neumark, J. Chem. Phys. 121, 6317 (2004)

${ }^{71}$ S.-H. Lee and K. Liu, J. Chem. Phys. 111, 6253 (1999).

${ }^{72}$ F. Dong, S.-H. Lee, and K. Liu, J. Chem. Phys. 115, 1197 (2001).

${ }^{73}$ N. Balucani, D. Skouteris, L. Cartechini, G. Capozza, E. Segoloni, P. Casavecchia, M. H. Alexander, G. Capecchi, and H.-J. Werner, Phys. Rev. Lett. 91, 013201 (2003).

${ }^{74}$ S.-H. Lee, K. Liu, and H. Chang, J. Chem. Phys. 110, 8229 (1999).

${ }^{75}$ J. O. Hirschfelder, H. Eyring, and B. Topley, J. Chem. Phys. 4, 170 (1936).

${ }^{76}$ S. Sato, J. Chem. Phys. 23, 2465 (1955).

${ }^{77}$ U. Manthe, W. Bian, and H.-J. Werner, Chem. Phys. Lett. 313, 647 (1999).

${ }^{78}$ X. A. Wang, W. Dong, C. Xiao, L. Che, Z. Ren, D. Dai, X. Wang, P. Casavecchia, X. Yang, B. Jiang, D. Xie, Z. Sun, S.-Y. Lee, D. H. Zhang, H.-J. Werner, and M. H. Alexander, Science 322, 573 (2008).

${ }^{79}$ N. Balucani, D. Skouteris, G. Capozza, E. Segoloni, P. Casavecchia, M. H. Alexander, G. Capecchi, and H.-J. Werner, Phys. Chem. Chem. Phys. 6, 5007 (2004).

${ }^{80}$ B. F. Parsons and D. W. Chandler, J. Chem. Phys. 122, 174306 (2005).

${ }^{81}$ Z. Sun, D. H. Zhang, and M. Alexander, J. Chem. Phys. 132, 034308 (2010).

${ }^{82}$ D. Skouteris, D. E. Manolopoulos, W. Bian, H.-J. Werner, L.-H. Lai, and K. Liu, Science 286, 1713 (1999).

${ }^{83}$ M. A. A. Clyne and D. H. Stedman, Trans. Faraday Soc. 62, 2164 (1966).

${ }^{84}$ A. A. Westenberg and N. Dehaas, J. Chem. Phys. 48, 4405 (1968).

${ }^{85}$ S. R. Leone and C. B. Moore, Chem. Phys. Lett. 19, 340 (1973).

${ }^{86}$ G. Y. Adusei and A. Fontijn, J. Phys. Chem. 97, 1409 (1993).

${ }^{87}$ F. J. Aoiz, B. Friedrich, V. J. Herrero, V. S. Rábanos, and J. E. Verdasco, Chem. Phys. Lett. 289, 132 (1998).

${ }^{88}$ F. J. Aoiz, V. J. Herrero, V. S. Rábanos, I. Tanarro, and J. E. Verdasco, Chem. Phys. Lett. 306, 179 (1999).

${ }^{89}$ F. J. Aoiz, L. Bañares, T. Bohm, A. Hanf, V. J. Herrero, K. H. Jung, A. Lłauter, K. W. Lee, M. Menéndez, V. S. Rábanos, I. Tanarro, H. R. Volpp, and J. Wolfrum, J. Phys. Chem. A 104, 10452 (2000).

${ }^{90}$ A. Läuter, R. K. Vatsa, J. P. Mittal, H. R. Volpp, and J. Wolfrum, J. Phys. Chem. A 110, 3273 (2006)

${ }^{91}$ P. M. Aker, G. J. Germann, and J. J. Valentini, J. Chem. Phys. 90, 4795 (1989).

${ }^{92}$ R. A. Brownsword, C. Kappel, P. Schmiechem, H. P. Upadhaya, and H. R. Volpp, Chem. Phys. Lett. 289, 241 (1998).

${ }^{93}$ V. J. Barclay, B. A. Collings, J. C. Polanyi, and J. H. Wang, J. Phys. Chem. 95, 2921 (1991).

${ }^{94}$ S. E. Branchett, S. B. Padkjær, and J. M. Launay, Chem. Phys. Lett. 208, 523 (1993).

${ }^{95}$ D. W. Schwenke, S. C. Tucker, R. Steckler, F. B. Brown, G. C. Lynch, D. C. Truhlar, and B. C. Garrett, J. Chem. Phys. 90, 3110 (1989).

${ }^{96}$ L. Yao, K. L. Han, H. S. Song, and D. H. Zhang, J. Phys. Chem. A 107, $2781(2003)$ 